\title{
Application of a New Method for Simultaneous Phase and Baseline Correction of NMR Signals (SINC)
}

\section{Ellen Steimers $^{1} \quad$ | Mathias Sawall ${ }^{2}$ | Richard Behrens ${ }^{1}$ \\ | Denise Meinhardt ${ }^{2,3}$ | Joël Simoneau ${ }^{4}$ | Kerstin \\ Münnemann $^{1}$ | Klaus Neymeyr ${ }^{2,3}$ | Erik von Harbou ${ }^{1}$}

\footnotetext{
${ }^{1}$ Technische Universität Kaiserslautern, Laboratory of Engineering Thermodynamics (LTD), Erwin-Schrödinger-Straße 44, 67663 Kaiserslautern, Germany

${ }^{2}$ Institute of Mathematics, University of Rostock, Ulmenstraße 69, 18057 Rostock, Germany

${ }^{3}$ Leibniz Institut für Katalyse, Albert-Einstein-Straße 29a, 18059 Rostock, Germany

${ }^{4}$ Université de Sherbrooke, Department of Chemical \& Biotechnological Engineering, 2500 Blvd. de L'Université, Sherbrooke, Canada

Correspondence

Erik von Harbou, Technische Universität Kaiserslautern, Laboratory of Engineering Thermodynamics,

Erwin-Schrödinger-Straße 44, 67663

Kaiserslautern, Germany

Email: erik.vonharbou@mv.uni-kl.de

Funding information Deutsche Forschungsgemeinschaft DFG (project numbers: 310714510 and 214012032)
}

Recently, we presented a new approach for simultaneous phase and baseline correction of NMR signals (SINC) that is based on multi-objective optimization. The algorithm can automatically correct large sets of NMR spectra, which are commonly acquired when reactions and processes are monitored with NMR spectroscopy. The aim of the algorithm is to provide spectra that can be evaluated quantitatively for example to calculate the composition of a mixture or the extent of reaction. In this work, the SINC algorithm is tested in three different studies. In an in-house comparison study, spectra of different mixtures were corrected both with the SINC method and manually by different experienced users. The study shows, that the results of the different users vary significantly and that their average uncertainty of the composition measurement is larger than the uncertainty obtained when the spectra are corrected with the SINC method. By means of a dilution study, we demonstrate that the SINC method is also applicable for the correction of spectra with low signal-to-noise ratio. Furthermore, a large set of NMR spectra that was acquired to follow a reaction was corrected with the SINC method. Even 
in this system, where the areas of the peaks and their chemical shifts changed during the course of reaction, the SINC method corrected the spectra robustly. The results show that this method is especially suited to correct large sets of NMR spectra and it is thus an important contribution for the automation of the evaluation of NMR spectra.

\section{KEYWORDS}

NMR spectroscopy, ${ }^{1} \mathrm{H}$ NMR,${ }^{13} \mathrm{C}$ NMR , Data processing, Simultaneous phase and baseline correction, Multi-objective optimization

\section{1 | INTRODUCTION}

Nuclear magnetic resonance (NMR) spectroscopy is an important technique for qualitative and quantitative analysis of complex multicomponent mixtures. As it features non-invasive measurements and enables quantitative evaluation of the measurements without prior calibration it is especially suited for online monitoring of reactions and processes 1, 1,5. In the frame of reaction and process monitoring, typically a large set of NMR spectra is acquired. When NMR spectroscopy is applied as a sensor for process control, continuously acquired NMR spectra have to be handled 6 . To be able to evaluate this large set of NMR spectra automatically and reliably, robust algorithms that require minimal input from the user are needed. Especially reaction monitoring is in this respect challenging as the composition of the mixture changes rapidly and as a result variations of the peak intensities and/or their chemical shifts (e.g. caused by variations of the $\mathrm{pH}$-value) are observed.

Reliable processing of the acquired NMR spectra is a prerequisite for their quantitative evaluation. This processing step includes (if carried out in the frequency domain) phase and baseline correction for each of the acquired NMR spectra. Only if these corrections are done properly, the accurate determination of the composition of the mixture via direct integration is possible. Malz et al. 7 showed the importance of proper phase and baseline correction and proofed the enormous influence of the user on the accuracy of the quantitative evaluation of NMR spectra. Furthermore, they described how the choice of the integration boundaries, which (if possible) should be 64 times the full width at half maximum (FWHM) value to cover $99 \%$ of the whole signal, influences the accuracy of the quantification results.

In order to minimize the influence of the user on the results and to simplify the processing of large sets of NMR spectra, many methods for automatic processing of NMR spectra have been published over the years, of which a few examples are presented here. Chen et al. 8 introduced the minimum entropy approach for the phase correction of NMR spectra. The phase corrected spectrum is determined by minimizing entropy, which is defined as the derivative of the normalized Fourier transformed NMR signals, in addition to a penalty function that ensures non-negative signals in the spectrum ${ }^{8}$. The baseline optimization method developed by Brown et al. 9 is based on the fact that a properly phased spectrum has a flat baseline with narrow peak bases. The phase and baseline corrected NMR spectrum is obtained by maximizing the number of real spectral data points within a defined baseline region with respect to the phase angles and the offset of the baseline. De Brouwer et al. 10 proposed a combined approach of baseline correction, area minimization and negative area penalization to obtain a robust and accurate method for the processing of Fourier transformed NMR signals, as usually only one of the requirements, either robust or accurate, is achieved. 
Area minimization is used as the robust function and area penalization, which ensures non-negative signals in the spectrum, is used to increase the accuracy of the results. Both, the robust and the accurate function are evaluated after a baseline correction is applied 10 .

Sawall et al. 11 described a new automatic approach for simultaneous phase and baseline correction of the Fourier transformed NMR signals that is based on a multi-objective optimization. As objective function they defined a weighted sum of a penalty function, which ensures non-negative signals in the spectrum and regularizing functions to account for sharp and smooth peaks. The approach differs from other approaches as the phase and the baseline are corrected simultaneously. The new approach is called SINC method (SImultaneous NMR Correction). Sawall et al. 11] showed the successful application of the SINC method for the simultaneous correction of the phase and the baseline of Fourier transformed NMR signals of a few simple samples.

In this paper, the application of the SINC method is tested in the field of reaction and process monitoring. First, the robustness of the method, which is the independence of the final (corrected) spectrum on the starting values of the phase angles (i.e. the uncorrected spectrum), is tested. Three subsequent experimental studies demonstrate the reliability and robustness of the SINC method for the processing of NMR spectra, which is a prerequisite for their quantitative evaluation. In an in-house comparison study, spectra of different samples with known compositions were corrected with the SINC method and evaluated quantitatively. In addition, eleven experienced users corrected the same spectra manually and used them to determine the composition of the samples. This test shows the accuracy of the SINC method and the independence from the user input. In a second experimental study, the SINC method was applied to spectra of a dilution series, to assess the performance of the method at low signal-to-noise ratios. In the last experimental study, we demonstrate that the SINC method is well-suited to correct a large set of NMR spectra reliably, which was taken from a kinetic study of the formation of methyl acetate. The results show that the performance of the SINC method makes it a valuable tool for processing of NMR signals and thereby it fosters a wider application of NMR spectroscopy for monitoring of reactions and processes.

\section{2 | THE SINC METHOD}

The algorithm of the SINC method is described in detail by Sawall et al. 11. Thus, only a brief description of the algorithm is given here. The main idea of the method is the simultaneous correction of the phase and the baseline of the Fourier transformed NMR signal. To do this, the algorithm uses multi-objective optimization to minimize the objective function, given by Equation 1. This objective function is a weighted sum of three different constraint functions that are applied to the normalized NMR spectrum.

$$
f(d)=\sum_{i=1}^{3} \gamma_{i} g_{i}\left(d /\|d\|_{\max }\right)
$$

Therein $d$ denotes a real-valued (discrete) spectrum, $\|d\|_{\max }$ is the maximum norm, and $\gamma_{i} \geq 0$ for $i=1,2,3$ are the weighting factors for the penalty / regularization terms. The function $g_{1}$ constrains negative signals in the spectrum. As the value of the weighting factor $\gamma_{1}$ is assigned to be much larger than the values of the weighting factors $\gamma_{2}$ and $\gamma_{3}$ the function $g_{1}$ is regarded as penalty function. Thus, the non-negativity of the signals in the spectrum is a stronger constraint. Due to the smaller values of their weighting factors, the functions $g_{2}$ and $g_{3}$ are considered as regularization functions, which ensure small integrals (characteristic for sharp and isolated peaks) and a smooth spectrum. By adjusting the values of the weighting factors, it is possible to account for different perturbations in the spectrum. In Sawall et al. 11, weighting factors of $\gamma_{1}=10, \gamma_{2}=0.1$ and $\gamma_{3}=0$ are applied for weakly disturbed spectra 
yielding good results. The algorithm starts with an initial phase correction of the Fourier transformed NMR signal $d^{\mathrm{ft}}$. The Fourier transformed NMR signal is related to the phase corrected NMR spectrum via the following equations.

$$
\begin{aligned}
& \operatorname{Re}\left(d_{j}^{\mathrm{pha}}\right)=\operatorname{Re}\left(d_{j}^{\mathrm{ft}}\right) \cos \left(\Phi_{j}\right)-\operatorname{Im}\left(d_{j}^{\mathrm{ft}}\right) \sin \left(\Phi_{j}\right) \\
& \operatorname{Im}\left(d_{j}^{\mathrm{pha}}\right)=\operatorname{Im}\left(d_{j}^{\mathrm{ft}}\right) \cos \left(\Phi_{j}\right)+\operatorname{Re}\left(d_{j}^{\mathrm{ft}}\right) \sin \left(\Phi_{j}\right)
\end{aligned}
$$

Therein $\operatorname{Re}\left(d_{j}^{\text {pha }}\right)$ and $\operatorname{Im}\left(d_{j}^{\text {pha }}\right)$ are the real and imaginary parts of the $j^{\text {th }}$ data point of the phase corrected NMR spectrum. $\operatorname{Re}\left(d_{j}^{\mathrm{ft}}\right)$ and $\operatorname{Im}\left(d_{j}^{\mathrm{ft}}\right)$ represent the real and imaginary parts of the $j^{\text {th }}$ data point of the uncorrected spectrum. The vector $\Phi_{j}$ is the total phase correction applied to the $j^{\text {th }}$ data point.

$$
\Phi_{j}=\varphi_{0}+\varphi_{1} \frac{j-1}{n}
$$

Herein $\varphi_{0}$ and $\varphi_{1}$ are the zero and first order phase angles. The total number of data points is given by $n$. The preliminary corrected NMR spectrum is obtained by minimizing the objective function (Equation 1]) with respect to the phase angles $\varphi_{0}$ and $\varphi_{1}$.

In a second step the pure baseline regions $M_{\mathrm{b}}$ are detected. A pure baseline region is referred to as a set of chemical shift values with intensities that are not assigned to any NMR signal. The detection of the pure baseline regions is crucial, as these regions should not include data points of a significant signal 11 . First, a Savitzky-Golay filter is applied to the preliminary corrected NMR spectrum to increase the signal-to-noise ratio, while maintaining the shape and the height of the peaks. Usually, a window length of 41 and a polynomial filter of degree one is applied, but can also be changed according to the data. Thereby, a smooth spectrum is obtained by a local least square polynomial approximation of the data points $\frac{12}{12}$. By a following robust cut-off selection, a set of indices is detected that belongs to the pure baseline regions 11 .

After the computation of the preliminary corrected spectrum and the detection of the pure baseline regions of this spectrum, a simultaneous optimization of the phase and the baseline starts. In each iteration of the simultaneous optimization, the phase corrected spectrum $d^{\text {pha }}$ and the baseline $u$ are computed. The baseline is computed with respect to the pure baseline regions. It is not necessary to recompute the set of indices of the pure baseline regions in every iteration as the changes in $M_{\mathrm{bl}}$ are expected to be very small 11]. The baseline is computed by applying the baseline recognition method proposed by Eilers 13 and Cobas et al. 14 , which has a similar performance as the Whittaker-smoother 11 . This method ensures that the baseline is a smooth function that approximates the data of the real part of the Fourier transformed NMR signal for the detected pure baseline regions. Finally, the objective function $\mathrm{f}\left(d^{\text {pha }}-u\right)$ (Equation 1] $)$ is minimized with respect to the phase angles $\varphi_{0}$ and $\varphi_{1}$. The minimization of the objective function is carried out in two steps. First, a genetic algorithm with a population size of 20 with 20 generations is used as predictor and then the final optimization is done by the ACM-routine NL2SOL ${ }^{15}$. The algorithm is implemented in MATLAB with the use of $C$ and Fortran. The algorithm corrects one spectrum at a time, using the previous result as the starting value for the following spectrum, which enables a parallelization. Therefore, the spectra can be splitted into blocks according to the number of kernels. 


\section{3 | MATERIALS AND METHODS}

\section{$3.1 \mid$ Chemicals 1}

Acetonitrile (ACN, 99.99 mass-\%) was purchased from Fisher Chemical. Methyldiethanolamine (MDEA, $\geq 99$ mass-\%), 1,4-dioxane (99.8 mass-\%), 2-propanol (Iso, $\geq 99.5$ mass-\%), and ethyl acetate (EtAc, $\geq 99.5$ mass-\%) were purchased from Sigma Aldrich. Toluene (Tol, $\geq 99.9$ mass-\%) and methanol ( $\mathrm{MeOH}, \geq 99.8$ mass-\%) were purchased from Merck KGaA. Sodium carbonate $\left(\mathrm{Na}_{2} \mathrm{CO}_{3}, \geq 99.8\right.$ mass-\%) was purchased from Th. Geyer. Acetic acid (AA, $\geq 99.8$ mass-\%) and sulfuric acid $\left(\mathrm{H}_{2} \mathrm{SO}_{4}, \geq 96\right.$ mass-\%) were purchased from Roth. Water was deionized and purified with a water purification system (Milli-Q Reference A+ System, Merck Millipore). Sodium carbonate was dried for $12 \mathrm{~h}$ at $120^{\circ} \mathrm{C}$ before using. All other chemicals were used without further purification.

\section{2 | Test of Robustness}

The robustness of the SINC method was determined using a mixture of toluene and 2-propanol (see Figure 3. The mixture was analyzed with ${ }^{1} \mathrm{H}$ NMR spectroscopy using a benchtop NMR spectrometer (Spinsolve Carbon, Magritek) equipped with a $1 \mathrm{~T}$ permanent magnet corresponding to a ${ }^{1} \mathrm{H}$ NMR Larmor frequency of $42.5 \mathrm{MHz}$. The corresponding acquisition parameters of the NMR measurement are given in Section 3.4 The minimum of the objective function is found to be located at the optimal phase angles of $\varphi_{0}^{*}=2.0986$ and $\varphi_{1}^{*}=-4.9870$. In order to test the robustness of the algorithm, a regularly spaced grid of starting values with 40 grid points in $\varphi_{0}$ direction and 30 grid points in $\varphi_{1}$ direction within the following intervals was used.

$$
\left(\varphi_{0}, \varphi_{1}\right) \in\left[\varphi_{0}^{*}-2.5 \pi, \varphi_{0}^{*}+2.5 \pi\right] \times\left[\varphi_{1}^{*}-1.5 \pi, \varphi_{1}^{*}+1.5 \pi\right]
$$

For every set of starting values the optimal phase angles were obtained by minimizing the objective function in Equation (1] using the two step optimization described in Chapter 2 The robustness of the SINC method is defined here as the percentage of optimization runs that yield the global minimum of the objective function $\left(\varphi_{0}^{*}, \varphi_{1}^{*}\right)$, after applying the two step optimization. In order to demonstrate the importance of the genetic algorithm as a predictor of the two step optimization, in a second test only the ACM-routine NL2SOL is used for the minimization of the objective function.

\section{3 | Experimental Procedure}

Three samples were prepared for the in-house comparison study. The compositions of the samples are listed in Table 1. All samples were gravimetrically prepared using a precision balance by Mettler Toledo (XS603S with an absolute uncertainty of $\pm 0.001 \mathrm{~g}$ specified by the manufacturer). By means of an uncertainty propagation, which included the uncertainty of the precision balance and the impurities of the chemicals, the mean of the absolute uncertainty of the gravimetrically determined mole fractions was estimated for every sample separately and is also given in Table 1 .

For the preparation of the dilution series, a precision balance by Mettler Toledo (PR2003 with an absolute uncertainty of $\pm 0.001 \mathrm{~g}$ specified by the manufacturer) was used. Sodium carbonate was dissolved in an aqueous stock solution of MDEA ( $w_{\text {MDEA }}=0.4 \mathrm{~g} \cdot \mathrm{g}^{-1}$ ). In total, 5 samples were prepared with mole fractions of sodium carbonate between $x_{\mathrm{Na}_{2} \mathrm{CO}_{3}}^{\mathrm{grav}}=0.01 \mathrm{~mol} \cdot \mathrm{mol}^{-1}$ and $x_{\mathrm{Na}_{2} \mathrm{CO}_{3}}^{\mathrm{grav}}=0.0001 \mathrm{~mol} \cdot \mathrm{mol}^{-1}$. By means of an uncertainty

\footnotetext{
${ }^{1}$ The values of the purities were adopted from the specifications given by the suppliers.
} 
propagation, the relative uncertainty of the gravimetrically determined mole fractions was estimated to be $0.1 \%$ for $x_{\mathrm{Na}_{2} \mathrm{CO}_{3}}<0.0005 \mathrm{~mol} \cdot \mathrm{mol}^{-1}$ and around $0.01 \%$ for $x_{\mathrm{Na}_{2} \mathrm{CO}_{3}}>0.0005 \mathrm{~mol} \cdot \mathrm{mol}^{-1}$ 16. For more details on the sample preparation, see Behrens et al. 16 .

The experimental set-up used for the kinetic study of the formation of methyl acetate is described in detail by Brächer et al. 217. The measurements were carried out using a micro-reactor NMR probe, which is well-suited for the monitoring of fast reactions (for details see 21718]). Thus, the time between mixing of the reactants (i.e. the start of the reaction) and analysis in the NMR flow cell is very short (less than two seconds) which allows monitoring of fast reactions 2 . The volumetric flow rates of the pumps were adjusted to ensure that the composition after the micro-mixer (i.e. at the beginning of the reaction) was an equimolar mixture of methanol $\left(x_{\mathrm{MeOH}}^{0}=0.4903 \mathrm{~mol} \cdot \mathrm{mol}^{-1}\right)$ and acetic acid $\left(x_{\mathrm{AA}}^{0}=0.4910 \mathrm{~mol} \cdot \mathrm{mol}^{-1}\right)$. In this work, the NMR probe was operated in the stopped flow mode. Thus, after steady state was reached, the flow was stopped and the composition of the reacting mixture in the NMR flow cell was monitored over time 2 .

\section{4 | NMR Analysis}

For the in-house comparison study, ${ }^{1} \mathrm{H}$ NMR spectra were acquired with the same benchtop NMR spectrometer that was used for the test of robustness mentioned above. The measurements were carried out with an acquisition time of $6.4 \mathrm{~s}$, a flip angle of $90^{\circ}, 64 \mathrm{k}$ data points, and 32 scans with a pulse repetition time of $5 \mathrm{~s}$.

The samples of the dilution series were measured with a high field NMR spectrometer with a $9.4 \mathrm{~T}$ vertical superconducting magnet corresponding to a ${ }^{1} \mathrm{H}$ NMR Larmor frequency of $400.25 \mathrm{MHz}$. The spectrometer is equipped with a probe whose electronics are cryogenically cooled (magnet Ascend 400, console Avance 3 HD 400, probe CryoProbe Prodigy, Bruker Biospin) $16 .{ }^{13} \mathrm{C}$ NMR measurements were carried out with a ${ }^{13} \mathrm{C}\left\{{ }^{1} \mathrm{H}\right\}$ inverse gated pulse sequence with a flip angle of $60^{\circ}$, a relaxation delay of $30 \mathrm{~s}$ and 512 scans.

In the kinetic study, the formation of methyl acetate was analyzed by ${ }^{1} \mathrm{H}$ NMR spectroscopy using the same high field magnet as described above. The NMR spectrometer is equipped with a micro-reactor probe ${ }^{2177}$. The ${ }^{1} \mathrm{H}$ NMR spectra were acquired with a flip angle of $10^{\circ}$ with a minimal pulse repetition time of $2 \mathrm{~s}$ and an acquisition time of $1 \mathrm{~s}$. Over a period of 75 minutes, 203 individual NMR spectra were acquired with pulse repetition times varying between $2 \mathrm{~s}$ and $60 \mathrm{~s}$. More details on the acquisition parameters for the three studies are given in the Supporting Information in Section $\mathrm{S.1}$

\section{5 | Data Evaluation}

For all three experimental studies, the weighting factors of the objective function (for details, see Section 2] used for the processing of the ${ }^{1} \mathrm{H}$ and ${ }^{13} \mathrm{C}$ NMR spectra with the SINC method, were adopted from Sawall et al. ${ }^{111}$. They found a set of weighting factors for the SINC method that gives good results for a large variety of phase and baseline deviations, typically observed in NMR spectra. By adjusting these weighting factors, the accuracy of the results could be further improved. The selection of the weighting factors should be done with respect to the data and its signal to noise ratio. In particular, the algorithm includes a threshold (a small negative value) for accepting slight negative entries of noise, see Hansen [19] for more details on the handling of noise. If the user is unsure about the results, an L-curve can be used 1920. An L-curve is helpful to balance two weights in a pareto-optimization. Therefore, the ratio of the two weights is varied. For every pair, the optimal solution is computed and the values of the individual penalty terms are plotted against each other. Typically, the plot has the shape of an $L$ and the weights according the kink correspond to a proper trade-off between regulations. 
The integration boundaries, applied to calculate the areas under the different peaks, were chosen by the coordinator of the studies. In the following, this evaluation procedure is called SINC.

The ${ }^{1} \mathrm{H}$ NMR spectra of the in-house comparison study were evaluated with the SINC method as described above. In addition, eleven experienced users from our laboratory evaluated the same spectra manually. For the manual processing of the ${ }^{1}$ H NMR spectra, the software MestReNova (MestReLabs, v12.01) was used. In order to eliminate the phase deviations, the two phase angles were adjusted manually according to the user's perception. For the baseline correction, the user could select from different algorithms provided by MestReNova.

The accuracy of the quantitative evaluation of NMR spectra does not only depend on the phase and baseline correction but also on the integration boundaries chosen for the determination of the areas under the peaks 7 . Thus, three different procedures were carried out to separate the influence of the phase and baseline correction from the influence of the choice of the integration boundaries on the analysis uncertainty. In procedure A, each user defined the integration boundaries and applied them to their manually corrected spectra. In procedure B, the coordinator of the study defined the integration boundaries for the spectrum of each mixture. These integration boundaries were applied to the manually corrected spectra of each user. This procedure ensures that the uncertainty of the quantitative evaluation of the spectra, introduced by the choice of the integration boundaries, is the same for all spectra. In procedure $\mathrm{C}$, the user defined integration boundaries were applied one by one to the spectra of the samples automatically processed with the SINC method, to see the influence of the choice of the integration boundaries on the results independent from the phase and baseline correction. Furthermore, automatic processing algorithms provided by MestReNova were used for the phase and baseline correction of the ${ }^{1} \mathrm{H}$ NMR spectra of the in-house comparison study. The areas under the peaks were determined with the integration boundaries chosen by the coordinator of the in-house comparison study (similar to procedure B), to ensure a better comparison to the results obtained with the SINC method. Table 2 gives an overview of the different evaluation procedures including the phase and baseline correction and the selection of the integration boundaries for the ${ }^{1} \mathrm{H}$ NMR spectra of the in-house comparison study. The processing parameters selected by the eleven users and details on the automatic algorithms used for the standard method are presented in the Supporting Information in Section 5.2

The mole fractions of the different species in the samples were determined directly from the ratio of the peak areas. Herein, all peaks that correspond to the same species were used for the determination of the mole fraction of that species in the mixture. As toluene, 2-propanol and ethyl acetate show more than one peak in the spectrum, six different combinations of peaks were used to calculate the composition of the two mixtures toluene-2-propanol and toluene-ethyl acetate (for details, see Supporting Information Section S.3. In total, 78 results were obtained for the composition of the two mixtures: six different combinations of peaks $\times$ (eleven users + SINC + Standard). For the mixture of acetonitrile and 1,4-dioxane, only one combination of peaks exists, as only two peaks are present in the ${ }^{1} \mathrm{H}$ NMR spectrum. Thus, only 13 results are obtained for the composition of the mixture of acetonitrile and 1,4dioxane. The difference between the mole fractions of the species determined from the ${ }^{1} \mathrm{H}$ NMR spectra and the mole fractions known from the sample preparation was taken as measure to assess the performance of the different evaluation methods (SINC method, manual, standard method).

The evaluation of the ${ }^{13} \mathrm{C}$ NMR spectra of the dilution series of $\mathrm{Na}_{2} \mathrm{CO}_{3}$ in aqueous MDEA solution were conducted in three ways: automatically with the SINC method, manually and automatically with MestReNova. The areas under the peaks of both automatically and the manually corrected spectra were determined by direct integration using the software MestReNova. The integration boundaries were chosen according to the guide lines given by Malz and Jancke 7 . More details on the processing parameters of the spectra of the dilution series are given in the Supporting Information in Section S.2. The mole fraction of $\mathrm{Na}_{2} \mathrm{CO}_{3}$ was determined from the peak areas of the $\mathrm{CO}_{3}^{2-}$ peak, which corresponds to $\mathrm{Na}_{2} \mathrm{CO}_{3}$, and of the $\mathrm{CH}_{3}$ group of MDEA. Additionally, the mass fraction of MDEA in the $\mathrm{Na}_{2} \mathrm{CO}_{3}$-free 
aqueous stock solution $W_{\text {MDEA }}$ was used for the evaluation. For more details on the evaluation of the mole fraction of $\mathrm{Na}_{2} \mathrm{CO}_{3}$, see Behrens et al. 16 .

In the kinetic study of the formation of methyl acetate, a series of $203{ }^{1} \mathrm{H}$ NMR spectra was acquired. These spectra were also evaluated in three ways: automatically with the SINC method, manually, and automatically with MestReNova (for details see Supporting Information Section S.2. The manual processing of the ${ }^{1} \mathrm{H}$ NMR spectra was performed separately for every spectrum to ensure properly phase and baseline corrected spectra. The integration boundaries applied to the series of ${ }^{1} \mathrm{H}$ NMR spectra were the same for both automatically and manually processed spectra. The mole fraction of methyl acetate was calculated from the peak area ratios for every spectrum, i.e. as a function of time. Because of partial overlapping, only the peaks of the $\mathrm{CH}_{3}$ group of methanol at about $3.2 \mathrm{ppm}$ and the $\mathrm{CH}_{3}$ group adjacent to the oxygen of methyl acetate, visible in the spectrum at about $3.4 \mathrm{ppm}$, were used for the quantitative evaluation of the spectra. Further details on the determination of the mole fractions of the reacting species are given in Brächer et al. 2 .

\section{4 | RESULTS AND DISCUSSION}

In order to demonstrate the performance of the SINC method, Figure 1 shows an ${ }^{1} \mathrm{H}$ NMR spectrum of an aqueous mixture of thiamine, pyridoxine, ascorbic acid, citric acid, malic acid, and glycin, measured on the benchtop spectrometer described above, before and after the phase and baseline correction with the SINC method. In addition, the figure shows a plot of the residuals between the processed and the unprocessed spectrum.

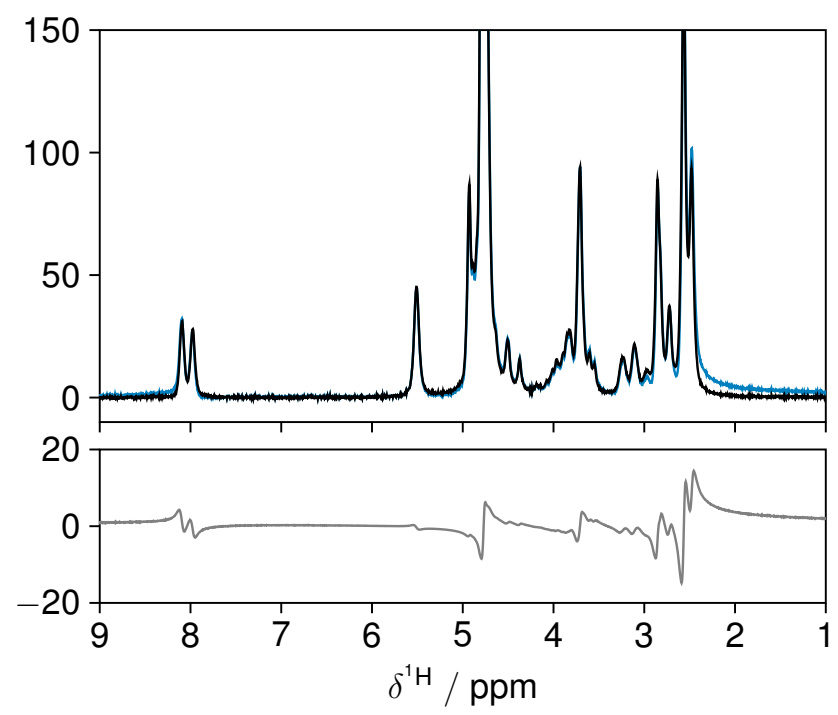

FIGURE $1{ }^{1}$ H NMR spectrum of an aqueous mixture of thiamine, pyridoxine, ascorbic acid, citric acid, malic acid, and glycin, unprocessed (blue) and after the simultaneous phase and baseline correction with the SINC method (black) (top) and a plot of the residuals (bottom).

In Figure 1 a well phased ${ }^{1} \mathrm{H}$ NMR spectrum is obtained after the simultaneous phase and baseline correction with the SINC method. This example demonstrates that the SINC method is even capable to process complex spectra 
with regions of many overlapping peaks, as it is the case in many biofluids (e.g. metabonomics).

\subsection{Robustness of the SINC Method}

Figure 2 shows the ${ }^{1} \mathrm{H}$ NMR spectrum of the mixture of toluene and 2-propanol before (blue) and after (black) the simultaneous phase and baseline correction using the SINC method and a plot of the residuals, which highlights the differences between the spectra. A very well phased and baseline corrected spectrum is obtained. Figure 3 depicts

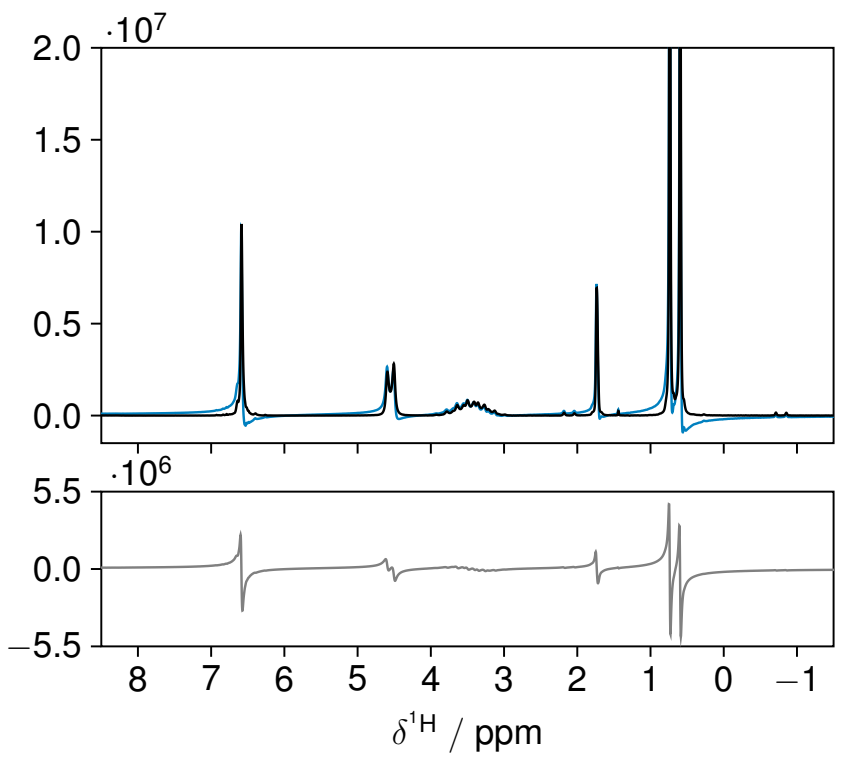

FIGURE $2{ }^{1}$ H NMR spectrum of toluene and 2-propanol unprocessed (blue) and after the simultaneous phase and baseline correction with the SINC method (black) (top) and a plot of the residuals (bottom).

the results of the test of robustness of the SINC method. On the left hand side the genetic algorithm is used as a predictor and on the right hand side the genetic algorithm is skipped, whereas the optimization algorithm is not changed in both plots. In Figure 3 lines connect the starting values with the optimized values. A blue line indicates a successful optimization otherwise the line is drawn black. The minima of the objective function are located at $\left(\varphi_{0}^{*}+2 m \pi, \varphi_{1}^{*}\right)$ with $m \in \mathbb{N}$. Using the genetic algorithm as predictor (cf. Figure 3 left) a robustness of $100 \%$ is achieved. Skipping the genetic algorithm (cf. Figure 3 right) results in a robustness of only $55.5 \%$. This result demonstrates the importance and functionality of the genetic algorithm as a predictor needed for a stable and successful optimization. The computation time for all $40 \times 30$ grid points (i.e. 1200 spectra) is $3756.60 \mathrm{~s}$ when using the proposed two step optimization and $2951.71 \mathrm{~s}$ when skipping the predictor (calculated on a standard PC with an intel-i7 processor with 3.4 GHz and $16 \mathrm{~GB}$ RAM using only one core). Thus, the predictor only slightly slows down the computation. The average computation time of the two step optimization is about $3 \mathrm{~s}$ per spectrum. The results show, that the SINC method robustly yields the optimal phase angles independent from the chosen start values of the phase angles (i.e. independent from the phase angles of the raw NMR spectrum) 2

\footnotetext{
${ }^{2}$ An interesting observation: For some optimizations the iteration converges not to the nearest minimum (all minima result in the same correction since the
} correction is $2 \pi$-periodic in $\varphi_{0}$ ). In such a case one or several iteration steps are too long and the iteration jumps into an adjacent catchment area. 

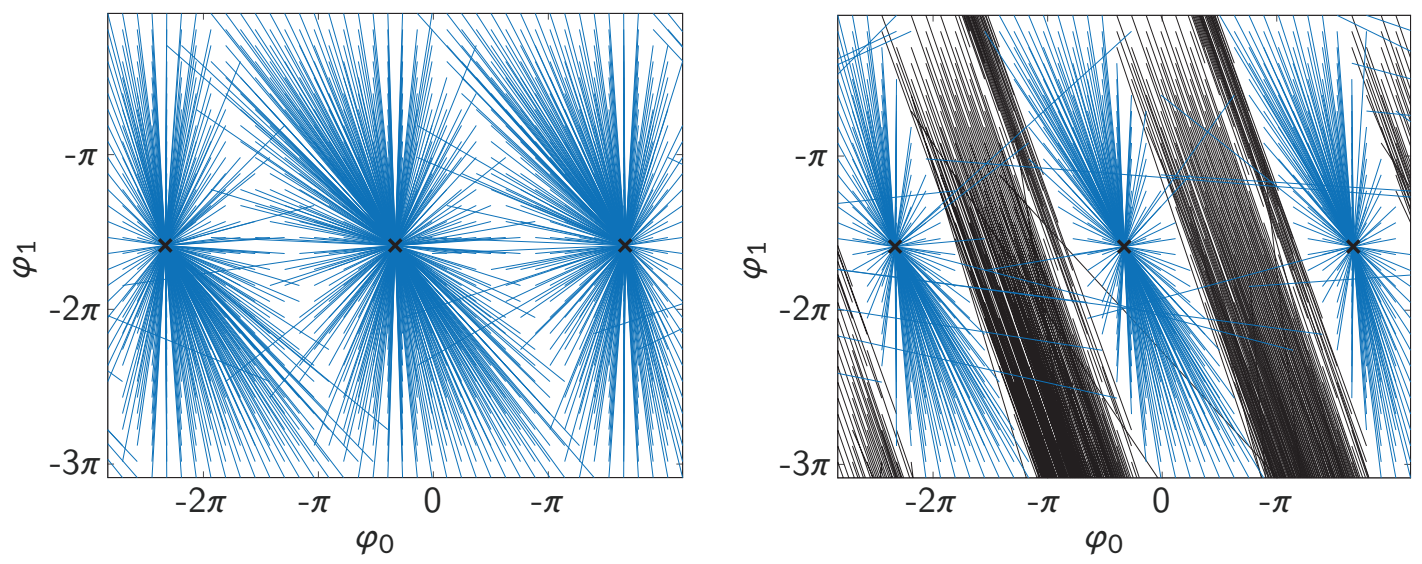

FIGURE 3 Robustness of the SINC method on a grid for the two phase angles $\varphi_{0}$ and $\varphi_{1}$, with (left) and without (right) the predictor (genetic algorithm). The starting centers (initial guesses) of the genetic algorithm are connected with the resulting minimum. A blue line indicates a successful optimization otherwise the line is drawn black.

\section{2 | In-House Comparison Study}

Figure 4 shows the results of the in-house comparison study. Each figure contains the results for the ${ }^{1} \mathrm{H} N \mathrm{MR}$ spectrum of one mixture that was quantitatively evaluated automatically with the SINC method, manually by the eleven users, and with the standard method.

Figure $4 a$ shows the results of the mixture of toluene-2-propanol, Figure $4 \mathrm{~b}$ shows the results of the mixture of toluene-ethyl acetate and Figure $4 \mathrm{c}$ shows the results of the mixture of acetonitrile-1,4-dioxane, respectively. In each figure, the different results of the mole fraction of one analyte are sorted from left to right starting with the lowest mole fraction values on the left side. Different symbols represent the different evaluation procedures. In addition, black lines show the mole fraction of the pertinent analyte that results from the gravimetric sample preparation. A surrounding gray box depicts the estimated uncertainty of the gravimetric sample preparation. The compositions obtained manually by the eleven users, scatter around the gravimetric values. In comparison to the users, the SINC method corrects the phase and minimizes baseline deviations in a more consistent manner so that the compositions scatter less than those determined by the user. The compositions obtained with the standard method scatter less than those obtained by the user but scatter more than those obtained with the SINC method. However, it should be noted that no optimization of the quantification procedure with the standard method was carried out as this was not in the scope of this study. The standard method was only used for a simple comparison. Figure 5 shows whisker plots of the relative uncertainties of the concentration measurement that compares the different evaluation procedures applied in the comparison study (for details see Table 2] with the SINC method. Both Figure 4 and Figure 5 show that even though some of the users achieved very small uncertainties in determining the composition (uncertainty less than $0.5 \%$, see Figure 5, others were far from the expected value (larger than $2 \%$, see Figure 5. At this point it is important to mention that all people who took part in the in-house comparison study were experienced NMR users. The variance of the quantification results and the deviation from the gravimetric value is largest for procedure $A$ (manual correction and free choice of the integration boundaries by the users). The contributions to the relative uncertainty caused by the manual correction of the spectra and the contribution caused by the choice of the integration boundaries are separated with the procedures $\mathrm{B}$ and $\mathrm{C}$ (for details see Table 2. When the spectra are corrected manually by the 
different users but the same integration boundaries as for the SINC method are applied (procedure B), the accuracy of the quantification increases compared to procedure A but the scattering of the results is still significantly larger than those found with the SINC method, as shown in the Figure 5 . The whisker plot for procedure $\mathrm{C}$ summarizes the quantification uncertainty for the case when the users chose only the integration boundaries but the correction of the NMR spectra is done with the SINC method. In procedures B and C, the median of the uncertainty is almost the same but in the latter case the scattering of the results is more significant. When the spectra are processed with the SINC method and the integration boundaries are chosen by the coordinator of the study (whisker plot on the right hand side of Figure 5 the scattering of the quantification uncertainty is significantly reduced (as shown by the reduction of the percentiles). Again, the larger uncertainty and the large noise of the quantification results found for procedure $A$ are caused by both the erroneous phase and baseline corrections and by the different choice of the integration boundaries of the users. In the current version of the SINC method, the integration boundaries have to be defined by the user. Thus, if the integration boundaries are not chosen carefully, also the SINC method can yield erroneous results. The comparison of the results obtained with procedure B and with the SINC method (see Figure 4) show, however, that already the improvement of the correction of the phase and baseline by the SINC method leads to significantly smaller uncertainties in the quantitative evaluation. These results of the comparison study demonstrate that manual phase and baseline correction as well as the manual selection of the integration boundaries cause significant scattering of the quantification results and highlight the necessity for a correction method like the SINC method that works robustly and independently from user inputs.

\section{3 | Dilution Series}

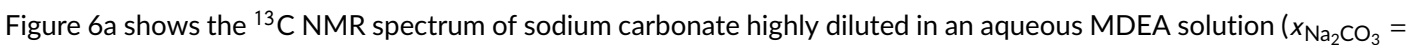
$\left.5 \cdot 10^{-4} \mathrm{~mol} \cdot \mathrm{mol}\right)$. Figure $6 \mathrm{~b}$ depicts the relative deviation between the mole fraction of sodium carbonate obtained from the gravimetric sample preparation and the mole fraction of sodium carbonate obtained from the ${ }^{13} \mathrm{C} \mathrm{NMR}$ spectra. Different symbols represent the results for the different evaluation methods of the spectra: automatic evaluation with the SINC method, manual evaluation, and automatic evaluation with the algorithms provided by MestReNova.

Figure $6 \mathrm{~b}$ shows that the relative deviations between the gravimetrically determined mole fractions of sodium carbonate and the mole fractions analyzed with ${ }^{13} \mathrm{C}$ NMR spectroscopy decreases with increasing signal-to-noise ratio (i.e. with increasing concentration of sodium carbonate). For the lowest investigated concentration of $\mathrm{Na}_{2} \mathrm{CO}_{3}$ $\left(x_{\mathrm{Na}_{2} \mathrm{CO}_{3}}=1 \cdot 10^{-4} \mathrm{~mol} \cdot \mathrm{mol}\right)$ the SINC method yields the lowest relative uncertainty of all three evaluation methods. However, even the SINC method is not capable to decrease the uncertainty to an acceptable level - simply because the NMR spectrometer reaches its limit for quantitative detection of ${ }^{13} \mathrm{C}$. For the higher concentrations of $\mathrm{Na}_{2} \mathrm{CO}_{3}$, the SINC method does not show a better performance than the other evaluation methods. Nevertheless, the study shows that the SINC method can be reliably applied to ${ }^{13} \mathrm{C}$ NMR spectra - even at low signal-to-noise ratios.

\section{4 | Kinetic Study}

Figure 7 shows a stacked plot of the ${ }^{1} \mathrm{H}$ NMR spectra that were acquired to follow the formation of methyl acetate. Because of the large number of spectra that has to be evaluated and because of the changes of peak height and position, this kind of data set is very challenging to evaluate. Hence, an automatic and robust evaluation method is favorable, which makes evaluating this data set an important test for the performance of the SINC method.

Figure 8 a shows one ${ }^{1} \mathrm{H}$ NMR spectrum acquired during the kinetic measurements of the formation of methyl acetate before (blue) and after (black) the automatic processing with the SINC method together with the plot of the 
residuals between the spectra. Once more, a very well phased and baseline corrected spectrum is obtained with the SINC method. Figure $8 \mathrm{~b}$ depicts the mole fraction of methyl acetate as a function of time, which is obtained after all ${ }^{1} \mathrm{H}$ NMR spectra were evaluated. Different symbols represent different evaluation methods: automatic evaluation with the SINC method, manual evaluation, and automatic evaluation with the algorithms provided by MestReNova. The differences between the mole fraction of methyl acetate (see Figure $8 \mathrm{~b}$ obtained with the different processing methods are not significant. Here, the benefit of the automatic processing with the SINC method, can not be found in smaller uncertainties but in the fact that no laborious, manual processing is needed. This study demonstrates the ability of the SINC method to process large sets of NMR spectra reliably.

\section{5 | CONCLUSIONS}

The different studies in this contribution show that the SINC method is well-suited for the automatic processing of NMR spectra. ${ }^{1} \mathrm{H}$ as well as ${ }^{13} \mathrm{C}$ NMR spectra are corrected robustly and reliably and the obtained quantitative results are at least as good as, if not better than the results after manual correction. Moreover, even at low signalto-noise ratios a good performance of the SINC method is confirmed. Furthermore, the SINC method enables a robust and automatic analysis of large sets of NMR spectra, acquired during reaction and process monitoring. At this point we would like to point out that only spectra with limited complexity have been investigated. In future work, the investigations should be extended to more complex spectra, e.g. to spectra with negative peaks (such as those obtained in DEPT experiments). The scope of this paper, however, was to demonstrate the ability of the algorithm to correct spectra acquired for reaction and process monitoring. For that reason, we optimized the algorithm to deal with standard 1D spectra.

By adjusting the weighting factors, the results obtained with the SINC method could be further improved. Additionally, it is possible to couple the SINC method with mathematical tools, that enable an automatic spectral analysis, e.g. the determination of concentrations of the analytes or the gathering of information on the process, such as reaction kinetics. Thus, the SINC method is a very useful tool for the quantification of NMR spectra that are acquired in the frame of reaction and process monitoring.

\section{acknowledgements}

The authors gratefully acknowledge financial support from the Deutsche Forschungsgemeinschaft (project numbers: 310714510 and 214012032).

\section{references}

[1] A. Brächer, L. M. Kreußer, S. Qamar, A. Seidel-Morgenstern, E. von Harbou, Chemical Engineering Journal 2018, 336 , 518-530.

[2] A. Brächer, R. Behrens, E. von Harbou, H. Hasse, Chemical Engineering Journal 2016, 306, 413-421.

[3] É. J. Kibrik, O. Steinhof, G. Scherr, W. R. Thiel, H. Hasse, Industrial \& Engineering Chemistry Research 2014, 53(32), 1260212613.

[4] N. Zientek, K. Meyer, S. Kern, M. Maiwald, Chemie Ingenieur Technik 2016, 88(6), 698-709.

[5] M. Maiwald, H. H. Fischer, Y.-K. Kim, K. Albert, H. Hasse, Journal of Magnetic Resonance 2004, 166(2), 135-146. 
[6] V. Sans, L. Porwol, V. Dragone, L. Cronin, Chemical Science 2015, 6(2), 1258-1264.

[7] F. Malz, H. Jancke, Journal of pharmaceutical and biomedical analysis 2005, 38(5), 813-823.

[8] L. Chen, Z. Weng, L. Goh, M. Garland, Journal of Magnetic Resonance 2002, 158(1-2), 164-168.

[9] D. E. Brown, T. W. Campbell, R. N. Moore, Journal of Magnetic Resonance (1969) 1989, 85(1), $15-23$.

[10] H. de Brouwer, Journal of Magnetic Resonance 2009, 201(2), 230-238.

[11] M. Sawall, E. von Harbou, A. Moog, R. Behrens, H. Schröder, J. Simoneau, E. Steimers, K. Neymeyr, Journal of Magnetic Resonance 2018, 289, 132-141.

[12] R. Schafer, IEEE Signal Processing Magazine 2011, 28(4), 111-117.

[13] P. H. C. Eilers, Analytical Chemistry 2003, 75(14), 3631-3636.

[14] J. C. Cobas, M. A. Bernstein, M. Martín-Pastor, P. G. Tahoces, Journal of Magnetic Resonance 2006, 183(1), $145-151$.

[15] J. E. Dennis Jr, D. M. Gay, R. E. Welsch, ACM Transactions on Mathematical Software (TOMS) 1981, 7(3), $369-383$.

[16] R. Behrens, E. von Harbou, W. R. Thiel, W. Böttinger, T. Ingram, G. Sieder, H. Hasse, Industrial \& Engineering Chemistry Research 2017, 56(31), 9006-9015.

[17] A. Brächer, S. Hoch, K. Albert, H. Kost, B. Werner, E. von Harbou, H. Hasse, Journal of Magnetic Resonance 2014, 242, 155-161.

[18] E. von Harbou, R. Behrens, J. Berje, A. Brächer, H. Hasse, Chemie Ingenieur Technik 2016, 89(4), 369-378.

[19] P. C. Hansen, SIAM Review 1992, 34(4), 561-580.

[20] H. W. Engl, M. Hanke, A. Neubauer, Regularization of inverse problems, Bd. 375 von Math. Appl., Kluwer Academic Publishers, Dordrecht, 2000.

TAB LE 1 Mole fractions and means of the absolute uncertainties of the gravimetrically prepared samples for the in-house comparison study

\begin{tabular}{|lllll|}
\hline sample & component & $x_{i} / \mathrm{mol} \cdot \mathrm{mol}^{-1}$ & absolute deviations $/ \mathrm{mol} \cdot \mathrm{mol}^{-1}$ & relative deviations $/ \%$ \\
\hline 1 & toluene & 0.2082 & \pm 0.0007 & 0.34 \\
\hline & 2-propanol & 0.7918 & \pm 0.0007 & 0.09 \\
\hline 3 & toluene & 0.4079 & \pm 0.0008 & 0.14 \\
\hline & ethyl acetate & 0.5921 & \pm 0.0008 & 0.21 \\
\hline & acetonitrile & 0.6799 & \pm 0.0006 & 0.08 \\
\hline
\end{tabular}


TABLE 2 Evaluation procedures for the quantitative analysis of the ${ }^{1} \mathrm{H}$ NMR spectra of the in-house comparison study.

\begin{tabular}{lrc} 
Procedure & Phase and baseline correction & Selection of integration boundaries \\
\hline A & manually by users & users \\
B & manually by users & coordinator \\
C & SINC & users \\
SINC & SINC & coordinator \\
Standard & automatic with MestReNova by coordinator & coordinator \\
\hline
\end{tabular}
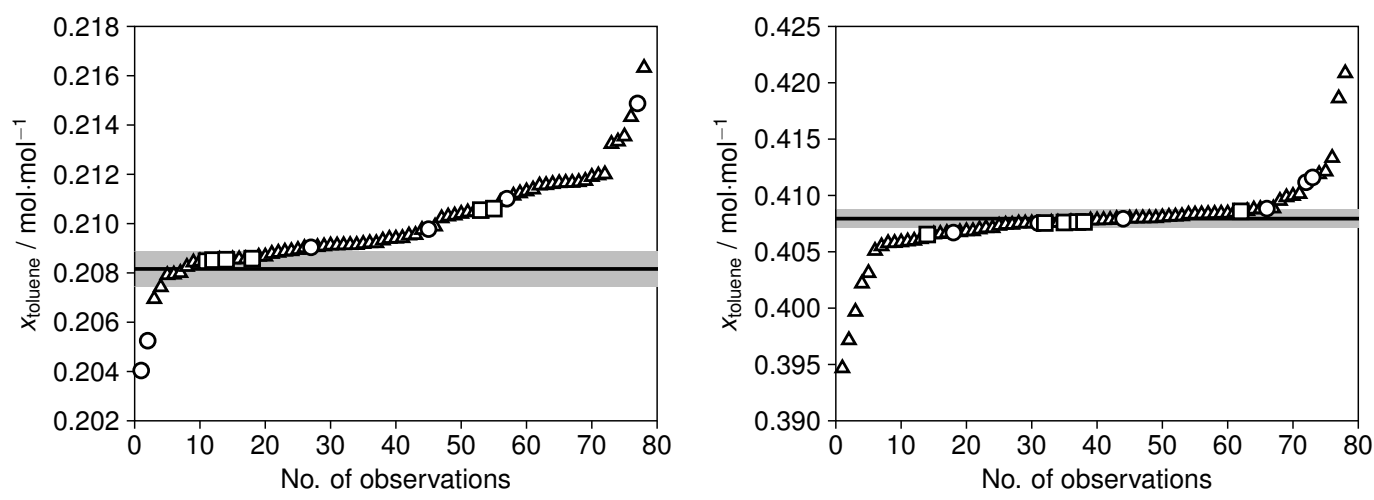

(a) Mixture of toluene $\left(x_{\text {toluene }}^{\text {grav }}=0.2082 \mathrm{~mol} \cdot \mathrm{mol}^{-1}\right)$ and 2-propanol. (b) Mixture of toluene $\left(x_{\text {toluene }}^{\text {grav }}=0.4079 \mathrm{~mol} \cdot \mathrm{mol}^{-1}\right)$ and ethyl acetate.

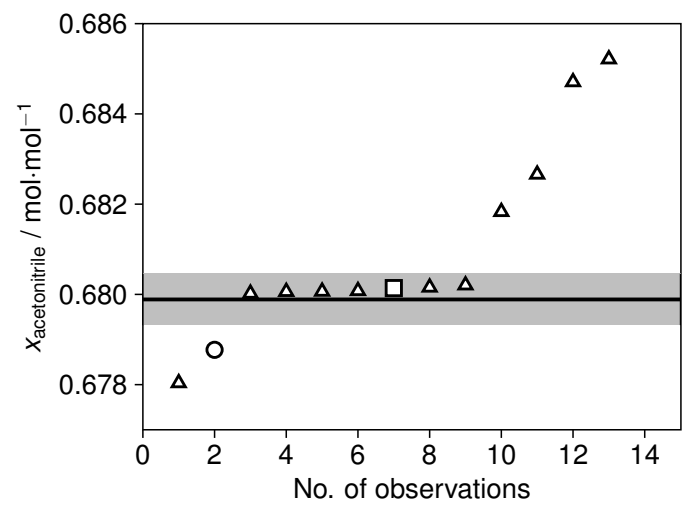

(c) Mixture of acetonitrile $\left(x_{\text {acetontrile }}^{\text {grav }}=0.6799 \mathrm{~mol} \cdot \mathrm{mol}^{-1}\right)$ and 1,4-dioxane.

FIGURE 4 Results of the in-house comparison study: mole fractions of the analytes in the three mixtures, obtained from the ${ }^{1} \mathrm{H}$ NMR spectra after automatic processing with the SINC method ( $\square$ ), manual processing by the different users (procedure $B$ ) $(\Delta)$, and with the standard method $(0)$. The solid lines represent the gravimetric values and the gray shaded area indicates the estimated uncertainty of the gravimetric sample preparation. 


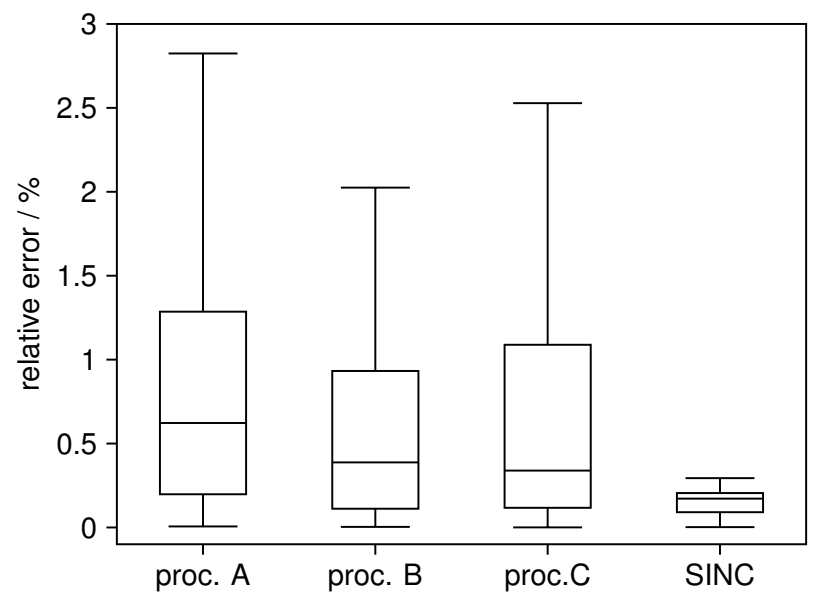

FIGURE 5 Whisker plot of the relative uncertainties of the mole fractions of the three analytes of the three samples shown in Figure 4 of the in-house comparison study. The relative uncertainties were determined from the gravimetric values and from the ${ }^{1} \mathrm{H}$ NMR spectra of the mixtures after the quantitative evaluation with the SINC method and by the users (procedure A-C) according to the evaluation procedures described in Table 2 The black solid line represents the median of the relative uncertainties, the edges of the boxes indicate the $25^{\text {th }}$ and $75^{\text {th }}$ percentiles and the most extreme data points are displayed by the whiskers. Outliers are not considered in the calculations of the whiskers and are omitted in this figure.

(a)

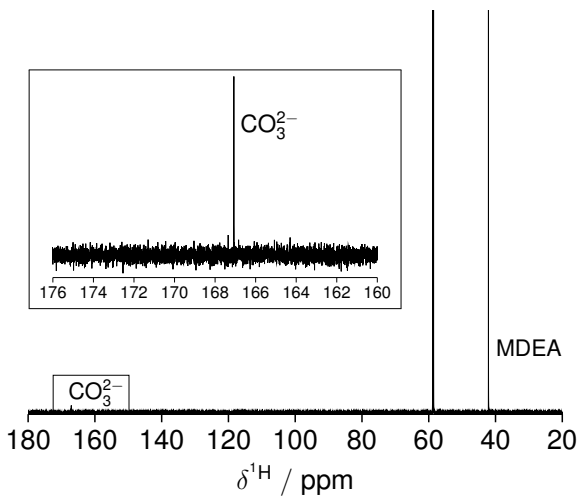

(b)

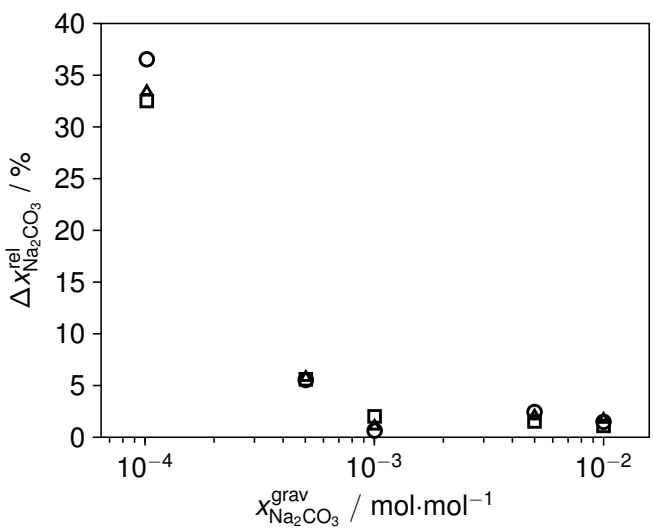

FIGURE 6 (a): ${ }^{13} \mathrm{C}$ NMR spectrum of sodium carbonate highly diluted in an aqueous MDEA solution $\left(x_{\mathrm{Na}_{2} \mathrm{CO}_{3}}=5 \cdot 10^{-4} \mathrm{~mol} \cdot \mathrm{mol}\right)$. (b): Comparison of the performance of different phase and baseline correction methods for ${ }^{13} \mathrm{C}$ NMR spectra of five samples of sodium carbonate highly diluted in an aqueous MDEA solution. The relative deviations between the mole fraction of sodium carbonate obtained from the gravimetric sample preparation and the mole fraction of sodium carbonate obtained from the ${ }^{13} \mathrm{C}$ NMR spectra, after automatic processing with the SINC method $(\square)$, manual processing $(\Delta)$, and with the automatic algorithms provided by MestReNova (O). 


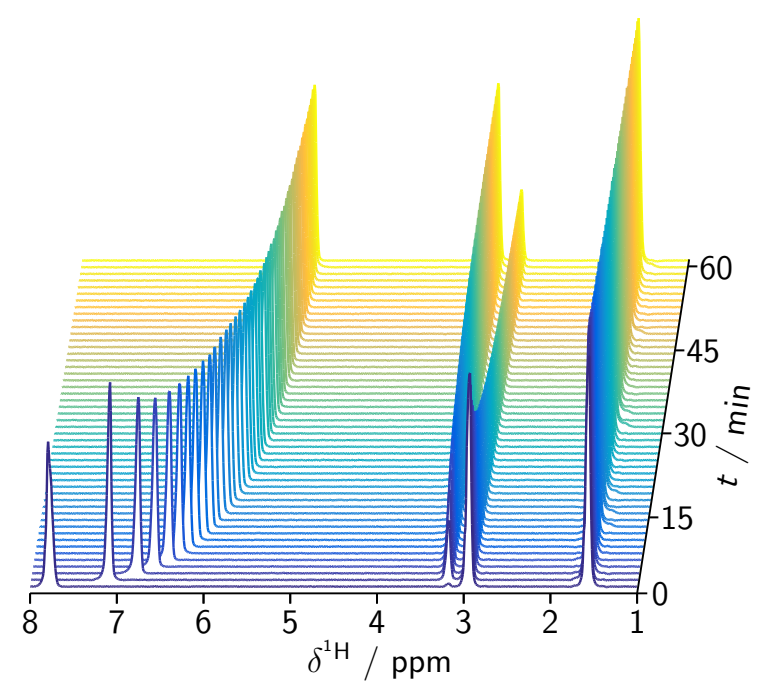

FIGURE 7 Stacked plot of the ${ }^{1}$ H NMR spectra acquired for the kinetic study of the formation of methyl acetate.

(a)

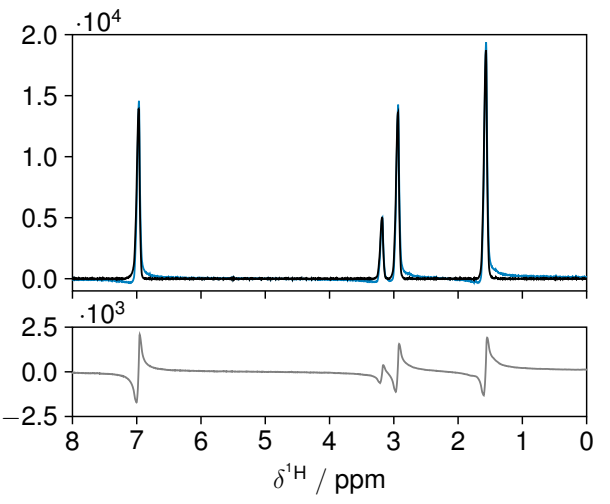

(b)

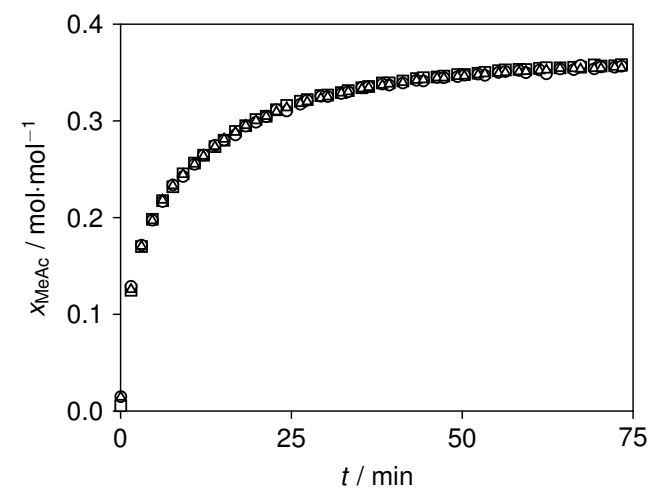

FIGURE 8 (a): ${ }^{1} \mathrm{H}$ NMR spectrum after an elapsed reaction time of 1.6 minutes during the formation of methyl acetate before (blue) and after (black) the simultaneous phase and baseline correction with the SINC method (top) and a plot of the residuals (bottom). (b): mole fraction of methyl acetate as a function of time, determined from the ${ }^{1} \mathrm{H}$ NMR spectra after automatic processing with the SINC method $(\square)$, manual processing $(\Delta)$, and automatic processing with the algorithms provided by MestReNova (O). 